\title{
CFD evaluation of thermal convection inside the DACON convection sensor in actual space flight
}

\author{
Pradyumna Ghosh, Mihir Kumar Ghosh
}

Department of Mechanical Engineering Institute of Technology, Banaras Hindu University (BHU), Varanasi, India; pradyumna_ghosh@rediffmail.com, $\underline{\text { mkghosh47@gmail.com }}$

Received 2 February 2011; revised 15 March 2011; accepted 27 March 2011.

\begin{abstract}
A CFD(Computational Fluid Dynamics) model has been developed using the commercial CFD package FLUENT for the thermal convection inside air filled cylindrical DACON sensor, where the onboard time dependent gravitational micro acceleration has been considered. Time dependent, curve fitted gravitational acceleration in $x$ - and $y$-axes from published data have been incorporated in FLUENT through a User Defined Function (UDF), developed in $C$ which includes space craft rotation. At the sensor plane the two-dimensional flow has also been visualized. A good agreement is between simulation and published experimental data. Last but not the least, for checking its response to sufficiently strong perturbations in an orbital flight, physical and numerical experiments are carried out where an astronaut swung the sensor in hands along the $y$ axis with amplitude of $10 \mathrm{~cm}$ and a frequency of $0.2 \mathrm{~Hz}$. A good qualitative validation has been achieved between CFD and actual experimental results.
\end{abstract}

Keywords: CFD (Computational Fluid Dynamics); DACON sensor; Experimental Data; FLUENT; UDF (User Defined Functions)

\section{INTRODUCTION}

Microgravity indicates low gravity, where the mean gravitational acceleration is in the range of $10^{-1}-10^{-5}$ $\mathrm{m} / \mathrm{s}^{2}$. All on-board experiments in the automatic and manned spacecraft experience residual micro-accelerations or g-jitter. These micro-accelerations are most significant in the frequency range $0-0.01 \mathrm{~Hz}$. Internal natural convection is a phenomenon of natural convection in an enclosure which has immense potential of engineering application. In this connection, DACON convection sensor has been developed to measure the re- sponse of micro-acceleration in the buoyancy driven thermal convection characteristics [1-5].

DACON convection sensor, shown in Figure 1, is an air-filled cylindrical cavity of strictly controlled boundary conditions for quick calculation of temperature field under real space flight conditions (under on-board micro-acceleration). The wall thickness of the cylinder is $2.5 \mathrm{~mm}$ and the thermal conductivity of the wall material is in the range of $0.16-2.3 \mathrm{~W} / \mathrm{m}-\mathrm{K}$. The junctions of the temperature sensors have been located $10 \mathrm{~mm}$ from the hotter isothermal wall at a diameter of $45 \mathrm{~mm}$, where the entire cylindrical curved surface is adiabatic [6].

These two crossed differential temperature sensor probes are placed in the cylindrical cavity in such a way that their sensitivity axis are perpendicular to the cylinder axis, where as constant temperature difference was maintained between the cylinder bases in actual space flight situation. In satellites and orbital stations micro-gravity oscillation has been felt as a consequence of spacecraft rotation, crew activity, different kind of vibration, non-uniformities of the earth's gravitational field etc. So far most of the studies have been concerned with the convection under constant reduced gravity or harmonic gravity vibration ( $\mathrm{g}$ jitter). However the actual behavior of fluid under orbital flight conditions is more complicated. Indeed, the space craft rotation not only contribute to the resultant vector micro-accelerations, but also gives rise to additional forces which cannot be reduced to some equivalent buoyancy forces.

In case of internal natural convection under sinusoidal g-jitter streaming flow has been observed where pulsating wave from hot and cold side travels towards the centre and engages in constructive/destructive interface to the formation of stationary wave [7].

The variable micro-acceleration due to rotation, angular acceleration and the gravity gradient can be expressed as [4-6],

$$
n=n a+\omega x(r x w)+r x \varpi+\kappa\left[3\left(e_{R} \cdot r\right) e_{R}-r\right]
$$

where $r$ is the radius vector of a point, $\omega$ is its angular 


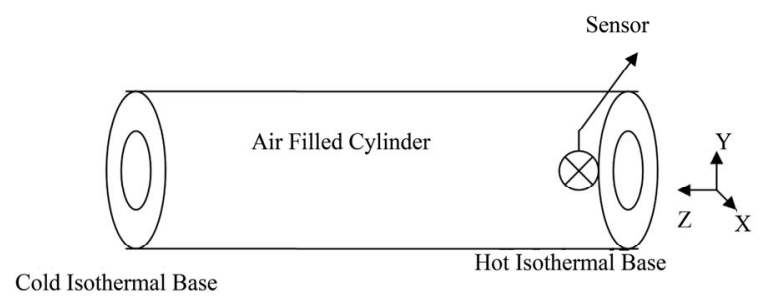

Figure 1. Description of DACON Sensor.

velocity, $\varpi$ is the angular acceleration, $e_{R}$ is the unit vector directed to the earth, $\kappa$ is the gravitational parameter, and $n_{a}$ is acceleration due to other causes(for example, spacecraft vibration) and $n$ is the variable micro-acceleration.

In the Boussinesq approximation, the buoyancy force can be expressed as,

$$
\rho n=\rho_{o} r x \varpi-\rho \beta\left(T-T_{o}\right) n
$$

where $T_{o}$ is mean temperature of the air, $\rho_{o}$ is the density at the temperature $T_{o}, \beta$ is the coefficient of thermal expansion. The first component of this force determines the action of angular acceleration, while second one only depends on relative temperature. Mathematical model has been presented as follows [6],

Transport equations:

$$
\nabla \cdot V=0
$$

$\rho\left[\frac{\partial V}{\partial t}+\nabla \bullet(V V)-2(V x \omega)\right]=-\nabla p+\nabla \cdot(\mu \nabla V)+\rho n$

$$
\rho C_{p}\left[\frac{\partial T}{\partial t}+\nabla \cdot(V T)\right]=\nabla \cdot(k \nabla T)
$$

Wall conduction:

$$
\rho_{w} C_{w} \frac{\partial T_{w}}{\partial t}=k_{w} \nabla^{2} T_{w}
$$

System of Eqs.3-6 has been described in the cylindrical system of co-ordinates $(\phi, z, r)$ in the region

$$
0 \leq \varphi \leq 2 \pi, 0 \leq z \leq L, 0 \leq r \leq R
$$

and for the shell wall $R \leq r \leq R_{w}$

For the velocities at all boundaries no-slip boundary conditions are used and initially the velocity throughout the cylinder has been considered as zero.

Temperature boundary conditions are as follows:

$$
\begin{gathered}
\left.T\right|_{z=0}=T_{h} \\
\left.\mathrm{~T}\right|_{z=L}=T_{l} \\
\left.T\right|_{r=R}=\left.T_{w}\right|_{r=R} \\
\left.k \frac{\partial T}{\partial r}\right|_{r=R}=\left.k_{w} \frac{\partial T_{w}}{\partial r}\right|_{r=R}
\end{gathered}
$$

$$
\left.\frac{\partial T_{w}}{\partial r}\right|_{r=R_{w}}=0
$$

where $V, p, T$ have been used for velocity, pressure and temperature respectively, $\rho, C_{p}, \mu, k$ have been symbolized for density, specific heat and viscosity and conductivity; ${ }_{w}$ (Subscript ) has been used for wall to consider the wall conduction effect.

Residual micro-acceleration are calculated for Mir station with space-craft's angular velocity, angular acceleration, gravity gradient in the center of mass of the station were calculated by V. V. Sazonov on the basis of telemetry data on the station orientation coming to the mission control center [3]. The data with a time step of 30s taken during the experiments onboard the Mir station in October, 1995 are used for calculating the combined micro-acceleration component $\mathrm{g}_{\mathrm{x}}$ and $\mathrm{g}_{\mathrm{y}}$, which has been described in Figure 2.

In the present investigation, commercial CFD package, FLUENT has been used to simulate the previous space flight experimental conditions or actual space flight experiment. CFD evaluation of the convection sensor's operation has been done with a semi numerical approach where real micro-acceleration data has been used from the reported data [6]. Then the CFD experiment has been performed to evaluate sensor temperature fluctuation with time, which has been also validated with the previously reported data. Moreover, with that confidence, stronger $g$ jitter perturbation for an astronaut swung in a Mir-station experiment has also been evaluated using CFD and compared with the actual experimental results. This approach (use of previously reported gravitational data) is more appropriate CFD modeling in this particular case. So normal numerical errors may result a large off-set with the experimental data and this procedure

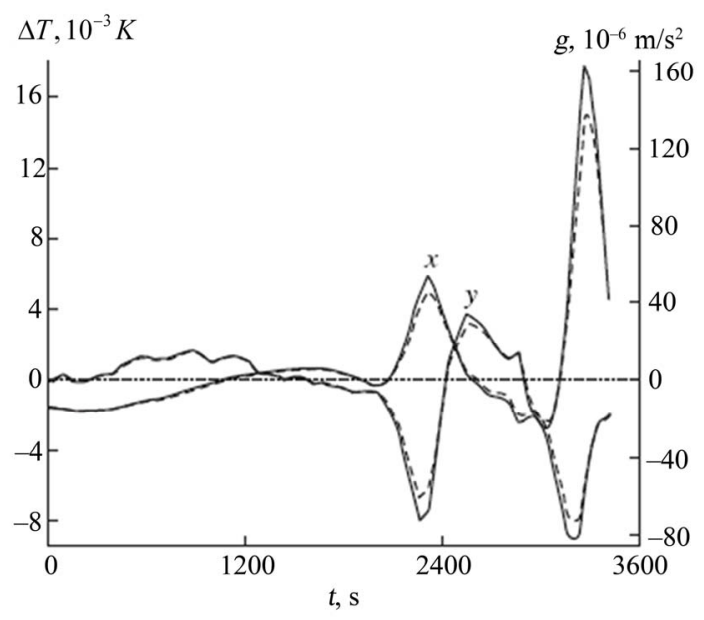

Figure 2. $g_{x}$ and $g_{y}$ and $T_{x}$ and $T_{y}$ from Previously Reported Results [3]. 
will reduce the probability of it, as one of the components of numerical calculation is pre verified. Thus CFD simulation provides more insight of the natural convection physics under actual g-jitter which had not been even captured through the physical experiment.

\section{CFD MODELING}

Firstly, an effort has been made to study the convection in the cylinder with hotter and colder isothermal bases with the entire adiabatic cylindrical curved surface, temperature difference in the thermocouple junctions under quasi-steady acceleration based on real microgravity field data. The CFD experiment has been exposed to real acceleration field. The cylindrical volume with a wall thickness of $2.5 \mathrm{~mm}$ as shown in Figure 3 has been finely meshed with tetrahedral volume meshes and four iso-point surfaces have been created in such a way that their sensitivity axis are perpendicular to the cylinder axis (z axis) to simulate the thermocouple junctions in GAMBIT. It has been observed that, mesh fineness increases the sensitivity of the sensor and as the DACON sensor has a very low sensitivity finest possible mesh has been used to capture the smaller fluctuation in scalar variables and eventually that increases the computation time.

These iso-point surfaces are located $10 \mathrm{~mm}$ away from the hot isothermal base at a radius of $45 \mathrm{~mm}$ as shown in Figure 4.

Like many other natural-convection flows, for faster convergence Boussinesq model has been used. This model treats density as a constant value (which has been supplied as the Material Property taking air as a working fluid) in all transport equations, except for the buoyancy term in the momentum equation. As the Boussinesq approximation is only valid when the temperature difference between the hotter and cooler wall is less where as for the present numerical experimentation that has been always considered as $50^{\circ} \mathrm{C}$ for air as working fluid $[1,6]$. All the relevant material properties for working fluid and

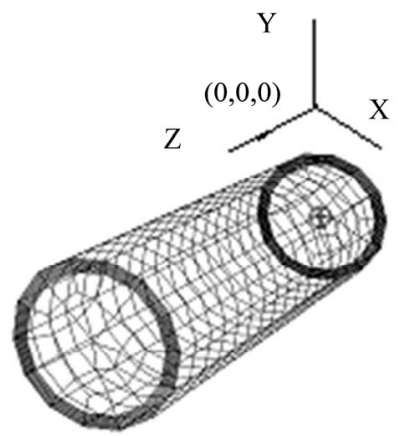

Figure 3. Meshed Cylindrical Air Filled Volume with Sensor Point Surfaces.

$$
z^{\frac{Y}{L}}
$$

Figure 4. Relative position of temperature sensor with respect to hotter isothermal cylindrical base.

Table 1. Thermophysical properties of the working fluid.

\begin{tabular}{ll}
\hline Property & Value \\
\hline Boussinesq Density & $0.001\left(\mathrm{~kg} / \mathrm{m}^{3}\right)$ \\
Specific Heat & $1.006(\mathrm{~kJ} / \mathrm{kg}-\mathrm{K})$ \\
Thermal Conductivity & $0.0242(\mathrm{~W} / \mathrm{m}-\mathrm{K})$ \\
Viscosity & $1.7894 \times 10^{-5}(\mathrm{~kg} / \mathrm{m}-\mathrm{s})$ \\
Molecular weight & $28.966(\mathrm{~kg} / \mathrm{kgmol})$ \\
Thermal Expansion Coefficient $(\beta)$ & $0.033(1 / \mathrm{K})$ \\
\hline
\end{tabular}

the shell conduction material have been described in Table 1.

Shell material conductivity has been considered 0.16 $2.3 \mathrm{~W} / \mathrm{m} . \mathrm{K}$. $\beta\left(T-T_{o}\right)$ term is going to be much lesser than 1 for this particular operating condition. So Boussinesq model has not been violated.

In the present investigation, constant gravitational acceleration has not been used as an operational condition, because gravitational acceleration in $x$ and $y$ axes are time dependent. A User Defined Function (UDF) has been developed in $\mathrm{C}$ to incorporate the time dependent variation in $g_{x}$ and $g_{y}$ (which includes space craft rotation, atmospheric drag etc) through the curve fitted equations from the published data $[3,6]$. The curve fitted time dependent combined micro-accelerations are as follows;

$$
\begin{aligned}
& \text { for } t<1200 \mathrm{~s} \\
& g_{x}=-2 E-11 t^{2}+3 E-08 t-4 E-20 \\
& g_{y}=1 E-11 t^{2}+3 E-10 t-2 E-05 \\
& \mathrm{R}^{2}=1
\end{aligned}
$$

and for $1200 \mathrm{~s}<t<3200 \mathrm{~s}$

$$
\begin{gathered}
g_{x}=-2 E-11 t^{2}+3 E-08 t-4 E-20 \\
g_{y}=3 E-15 t^{2}-3 E-11 t^{3}+1 E-07 t^{2}-0.0002 t+0.116 \\
\mathrm{R}^{2}=1
\end{gathered}
$$

where $t$ is time in sec and $g$ is $\mathrm{m} / \mathrm{s}^{2}$ and where $R^{2}$ is the regression coefficient.

This UDF has been used to modify the $x$ momentum 
and $y$ momentum equations as source terms in fluid boundary conditions. Two isothermal bases and adiabatic cylindrical curved surface have been declared as wall boundary. Hot isothermal wall temperature and cold isothermal wall temperatures are $298.15 \mathrm{~K}$ and $348.15 \mathrm{~K}$ respectively. Shell conduction has also been incorporated for more realistic model. More than 50,000 tetrahedral meshes have been used and all the results presented in this paper are mesh independent. Transient model has been used to solve the transport equations with a time step of $30 \mathrm{~s}$ and sensor temperature at the four junctions has been recorded at every time step. Convergence criterion for residuals has been selected as $10^{-6}$. However, much lower number of meshes $(35,000$ and 16,000) have also been used especially for astronaut experiment to verify the closeness of the numerical results with the experimental results.

Secondly, as the sensitivity of the DACON is relatively low, for checking its response to sufficiently strong perturbations in an orbital flight, an experiment is carried out where an astronaut swung the sensor in hands along the y axis(according to Figure 1) with an amplitude of $10-25 \mathrm{~cm}$ and a frequency of $0.18-0.2 \mathrm{~Hz}$ [6]. The same experiment has been carried out numerically modifying the gravitational UDF, just adding the additional perturbation in the $\mathrm{g}_{\mathrm{y}}$, where other conditions will be same as the previous numerical study.

\section{RESULTS AND DISCUSSION}

It has to be noted that, $\mathrm{x}$ axis, in previously reported results has to be considered as y axis in CFD experimental result and vice-versa. Curve fitted $g_{x}$ and $g_{y}$ with time has been plotted in Figure 5. Figure 6 indicates the temperature difference and $\mathrm{x}$ and $\mathrm{y}$ directional thermocouples sensors, $\mathrm{T}_{\mathrm{x}}$ and $\mathrm{T}_{\mathrm{y}}$ with time from the CFD experiment. Previous reported, $g_{x}$ and $g_{y}$ and $T_{x}$ and $T_{y}$ with time has been described in Figure 2, which indicates a good qualitative and quantitative match with the CFD experiment and previously reported results. It can be observed from the Figure 6 and Figure 2 that the sensor sensitivity is equal to approximately $10^{-3}$ degree per $10^{-5} \mathrm{~m} / \mathrm{s}^{2}\left(10^{-6} \mathrm{~g}_{0}\right)$. Using air as working fluid (due to comparatively low specific heat and no phase change problem) the sensor has a good time response. Even in the regions of strong variation of acceleration the time response of the sensor is about $3 \mathrm{~s}[3,6]$.

Figures 7-10 describe Temperature isotherms and velocity contours at sensor parallel plane at three different time steps where there is significant change in gravitational acceleration. Sensor parallel plane is comprised of three such points $[(0.0225 \mathrm{~m}, 0,0.012 \mathrm{~m}),(0,0.0225$, $0.012),(0,-0.0225,0.012)]$. Velocity magnitude and temperature contours have plotted at $390 \mathrm{~s}$ where both $\mathrm{g}_{\mathrm{x}}$
Variation of Gravitational Field with Time in Mir Space Station. [Oct.1995]

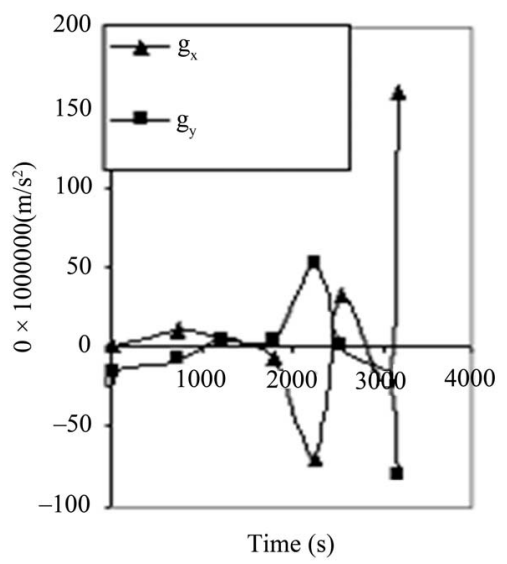

Figure 5. Curve-fitted Variation of Gravitational Field with Time in Mir Space Station (1995) [3].

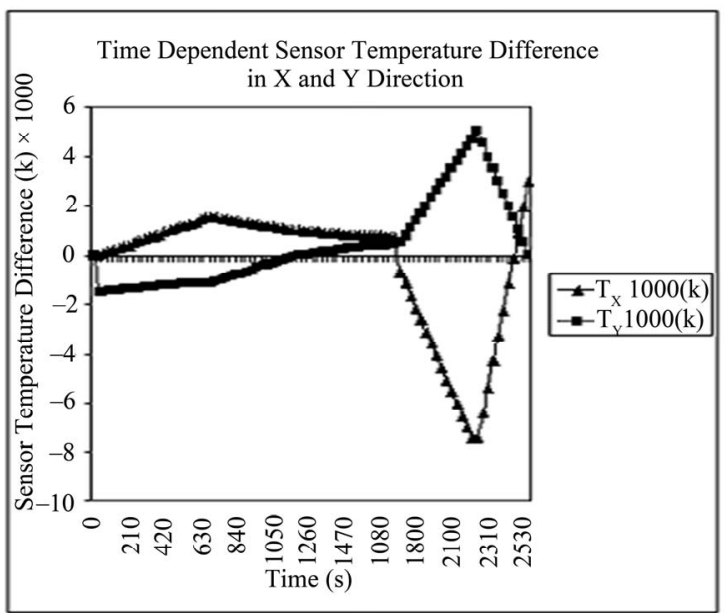

Figure 6. $T_{x}$ and $T_{y}$ from CFD Experiment in DACON Sensor using Previously Reported time Dependent Gravitational Field.

and $g_{y}$ are both are increasing initially, $1200 \mathrm{~s}$ where both $g_{x}$ and $g_{y}$ are both are almost equal and $2250 \mathrm{~s}$ where $g_{x}$ and $g_{y}$ are at respective crest and peak from the $g$ data. The rationale for the selection of these time steps is to investigate the variation in the flow pattern with the variation of $\mathrm{g}$.

Figure 7 describes the temperature isotherms on the sensor Plane at $390 \mathrm{~s}$. It has been observed that, temperature isotherms are almost static with time on the sensor plane as the temperature fluctuation at the sensor junctions with time is the order of $10^{-3}$ degree where as the global temperature range of the isotherms are of one degree.

Figure 7(b) shows the two different diffusion eddy zone in $y$ direction where $g_{x}$ is positive and $g_{y}$ is nega- 
$3.48 \mathrm{e}+02$ $3.45 \mathrm{e}+02$ $3.43 \mathrm{e}+02$

$3.40 \mathrm{e}+02$

$3.38 \mathrm{e}+02$

$3.35 \mathrm{e}+02$

$3.33 \mathrm{e}+02$

$3.30 \mathrm{e}+02$

$3.28 \mathrm{e}+02$

$3.25 \mathrm{e}+02$

$3.23 \mathrm{e}+02$

$3.20 \mathrm{e}+02$

$3.18 \mathrm{c}+02$

$3.15 \mathrm{e}+02$

$3.13 \mathrm{e}+02$

$3.10 \mathrm{e}+02$

$3.08 \mathrm{e}+02$

$3.05 \mathrm{e}+02$

$3.03 e+02$

$3.00 \mathrm{e}+02$

$2.98 \mathrm{e}+02$

(a)

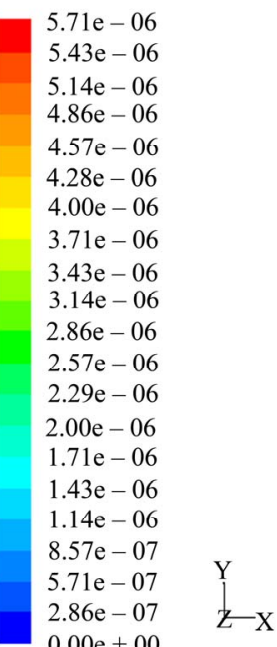

(b)

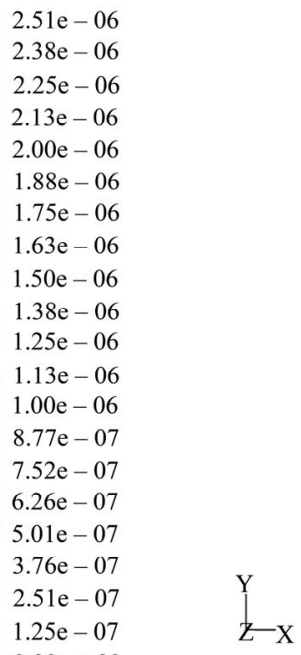

$0.00 \mathrm{e}+00$

(c)

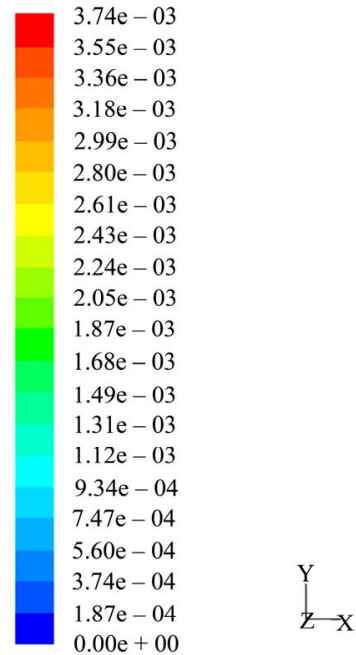

(d)

Figure 7. (a) Isotherms at the Sensor Plane $[(0.0225 \mathrm{~m}, 0$, $0.012 \mathrm{~m}),(0,0.0225,0.012),(0,0.0225,0.012)]$ at $390 \mathrm{~s}$; (b) Velocity Contours at Sensor Plane $[(0.0225 \mathrm{~m}, 0,0.012$ $\mathrm{m}),(0,0.0225,0.012),(0,-0.0225,0.012)]$ at $390 \mathrm{~s}$; (c) Velocity Contours at Sensor Plane $[(0.0225 \mathrm{~m}, 0,0.012 \mathrm{~m})$, $(0,0.0225,0.012),(0,-0.0225,0.012)]$ at $1200 \mathrm{~s}$; (d) Velocity Contours at Sensor Plane $[(0.0225 \mathrm{~m}, 0,0.012 \mathrm{~m}),(0$, $0.0225,0.012),(0,-0.0225,0.012)]$ at $2250 \mathrm{~s}$.

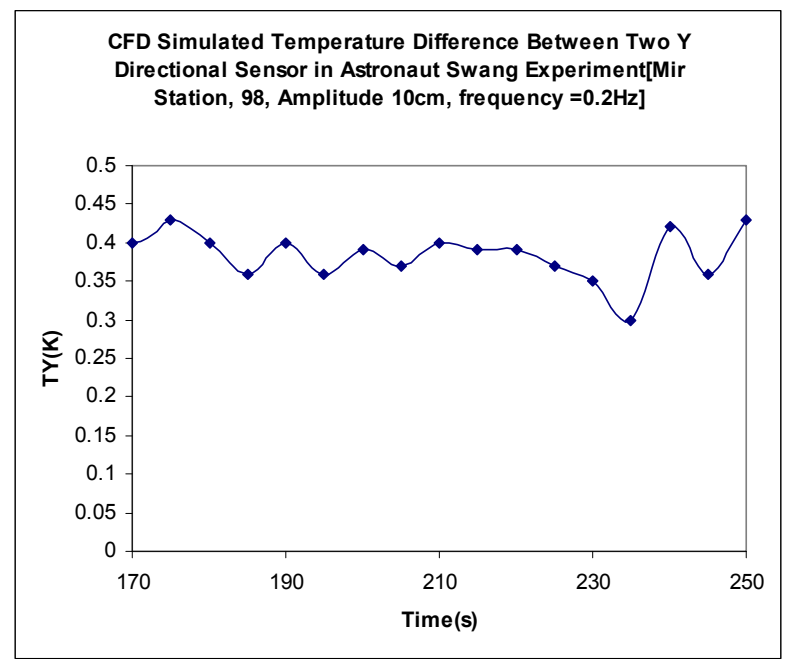

Figure 8. CFD Simulated Temperature Difference between Two Y Directional Sensor junctions in Astronaut Swung Experiment [Mir Station, 98, Amplitude $10 \mathrm{~cm}$, frequency $=0.2$ $\mathrm{Hz}$.

Tive (according to Figure 4) but with time $(t=1200 \mathrm{~s})$ it concentrates in negative $y$ direction as both the $g_{x}$ and $g_{y}$ are almost zero as shown in Figure 7(c). So there is very low intensity of diffusion. The velocity magnitude decreases by almost by 50 percent. It can be said that the 


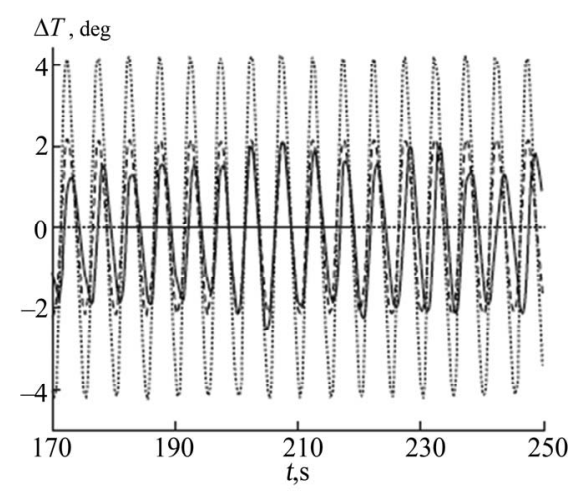

Figure 9. Temperature Difference Between Two Y Directional Sensor junctions in Astronaut Swung Experiment [Mir Station, 98, Amplitude 10,20 cm, frequency $=0.2 \mathrm{~Hz}$, Solid Line Experimental, Dotted Simulation result] [6].

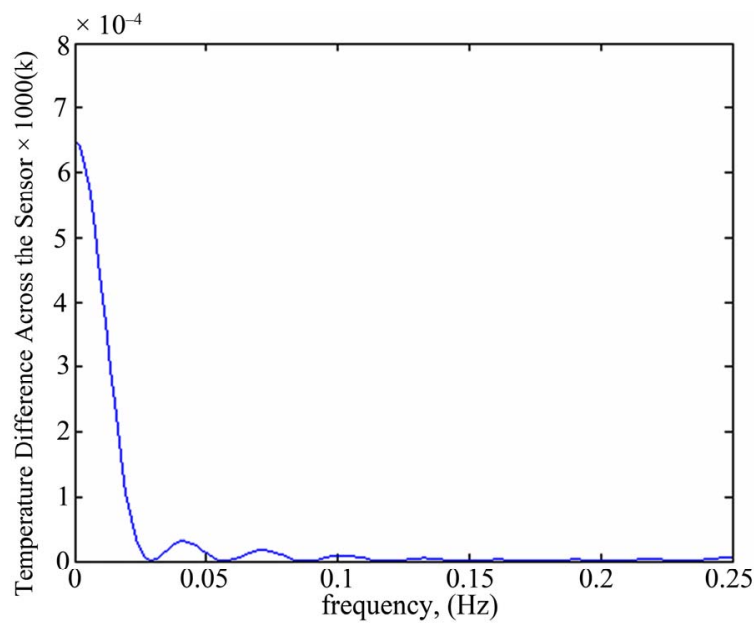

Figure 10. FFT of CFD Simulated Temperature Difference between Two Y Directional Sensor junctions in Astronaut Swung Experiment.

flow is basically based on thermal creep. At $(t=2500 \mathrm{~s})$ both the $g_{x}$ and $g_{y}$ are at their respective peak and crest, which increases the diffusion, and very small diffusion eddy zone has been developed in the positive quadrant of the sensor plane. The velocity magnitude increases almost by 1000 times as shown in Figure 7(d).

Numerical Experiment has been performed with a sufficiently strong perturbation in an orbital flight, where an astronaut swung the sensor in hands along the y axis (according to Figure 1) with amplitude of $10 \mathrm{~cm}$ and a frequency of $0.2 \mathrm{~Hz}$. This strong perturbation has been added vectorially with the actual gravitation field in $\mathrm{Y}$ direction whereas the $\mathrm{X}$-directional gravitational field has been unaltered. The temperature difference in $\mathrm{Y}$ directional sensor from $170 \mathrm{sec}$ to $250 \mathrm{sec}$ with a time interval of $5 \mathrm{sec}$ has been described in Figure 8, where it shows kind of sinusoidal oscillation like the previously reported data, shown in Figure 9 [6]. But the amplitude and frequency of oscillation does not match quantitatively with the reported results. But as the convection maximum velocity magnitude is increasing almost 1000 times it is kind of expected that magnitude of the reading in the sensor will also increase. The difference with the previous experimental results may be due to sensor sensitivity in that particular case. But CFD simulation results also did not match so well, quantitatively with the previous simulation, which had coarser grids $(64 \times 32 \times$ 16) in comparison with FLUENT simulation, where 50,000 tetrahedral meshes have been used. However, much lower number of meshes $(35,000$ and 16,000$)$ have also been used especially for astronaut experiment to verify the closeness of the numerical results with the experimental results but quantitatively CFD results and experimental or previously carried out numerical experimentation didn't match well. However, FFT analysis has been carried out with the response of the astronaut experiment as shown in Figure 10 which indicate that dominating frequency in the temperature response is about $0.04 \mathrm{~Hz}$ and $0.075 \mathrm{~Hz}$ which is different than the excitation frequency $0.2 \mathrm{~Hz}$ which is quite obvious for the nonlinear system response. Moreover, the velocity contour has also been plotted in Figures 11-12 for the sensor plane which indicates that the maximum velocity is almost $10^{3}$ higher than for the real microgravity field which has been presented in Figures 7(b)-7(d). It seems there is some missing fluid physics in case of astronaut swung experiment which has not been mentioned in [6].

\section{CONCLUSIONS}

The CFD simulation of DACON sensor has been do-

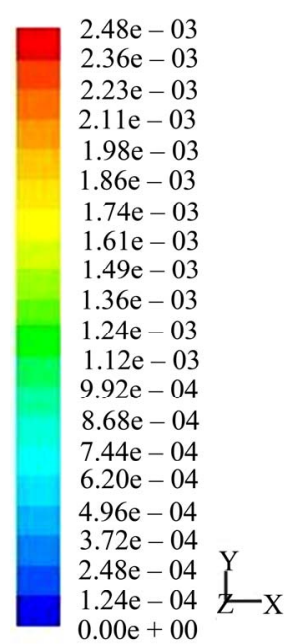

Figure 11. Velocity Contours at Sensor Plane $[(0.0225 \mathrm{~m}$, $0,0.012 \mathrm{~m}),(0,0.0225,0.012),(0,-0.0225,0.012)]$ during astronaut experiment at $170 \mathrm{~s}$. 


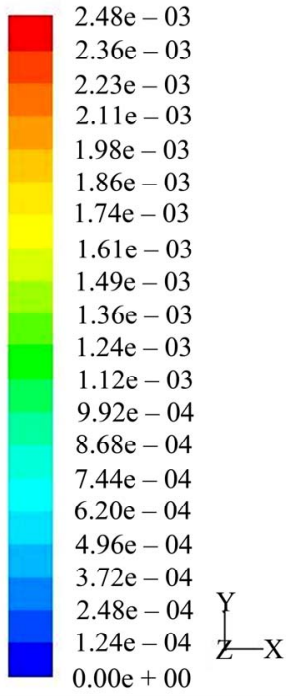

Figure 12. Velocity Contours at Sensor Plane $[(0.0225 \mathrm{~m}, 0$, $0.012 \mathrm{~m}),(0,0.0225,0.012),(0,-0.0225,0.012)]$ during astronaut experiment at $230 \mathrm{~s}$.

cumented in this present investigation. A good qualitative and quantitative match with the CFD experiment and previously reported results has been achieved for temperature fluctuation in the sensor with time. Transient nature of flow has been described, which clearly indicates the diffusion rate change and formation of diffusion eddy at the sensor plane with time due to thermal creep. Last but not the least, another CFD experiment has been performed with more strong perturbation and the results for the temperature fluctuation in sensor matches qualitatively with the previous experimental or numerical results, but there are quantitative differences. CFD results for the temperature response and its FFT analysis clearly indicate that other sub-harmonics are present in the response which is expected from such non-linear system response. The difference with the previous experimental results may be due to some other missing factors, which have not been reported, in the published literature, in that particular case. Moreover, CFD evaluation unfolds better insight of micro gravity convection and costly space experiment can be avoided if real micro gravity field is known from the previous experience.

\section{REFERENCES}

[1] Bogatyryov, G.P., Putin, G.F., Ermakov, M.K., et al., (1995) A System for analysis and measurement of convection aboard space station: objectives, mathematical and ground-based modeling. 33rd Aerospace Science Meeting and Exhibit, Reno, 1-10.

[2] Polezhaev, V.I., Ermakov, M.K., Nikitin, N.V., and Nikitin, S.A. (1997) Nonlinear interactions and temperature oscillations in low prandtl melt of czochralski model: validation of computational solutions for gravity-driven and rotatory flows. Proceedings of International Symposium On Advances in Computational Heat Transfer, Cesme, 26-30 May 1997. doi:10.1615/ICHMT.1997.IntSymLiqTwoPhaseFlowTran spPhenCHT.570

[3] Sazonov, V.V., Komarov, M.M., Polezhaev, V.I., et al., (1997) Microaccelerations on board the mir orbital station and quick analysis of the gravitational sensitivity of convective heat/mass transfer processes. Cosmic Reasearch, 35.

[4] Yuferev, V.S., Polovko, Y.A. and Sazonov, V.V. (1998) Three-dimensional thermal convection caused by spacecraft rotation in a rectangular enclosure with rigid walls Physics of Fluids, 10, 2517-2524. doi:10.1063/1.869767

[5] Babushkin, I.A., Bogatyrev, G.P., Glukhov, A.F., et al., (2001) Investigation of Thermal Convection and LowFrequency Microgravity by the $D A C O N$ Sensor aboard the Mir Orbital Complex. Cosmic Research, 39, 150-158.

[6] Bessonov, O.A. and Polezhaev, V.I. (2001) Mathematical modeling of convection in the dacon sensor under conditions of real space flight. Cosmic Research, 39, 170-178.

[7] Ghosh, P. and Ghosh, M.K. (2009) Streaming flows in differentially heated square porous cavity under sinusoidal g-jitter. International Journal of Thermal Sciences, 48, 514-520. doi:10.1016/j.ijthermalsci.2008.04.002 